\title{
RNA-binding protein Ptbp1 regulates alternative splicing and transcriptome in spermatogonia and maintains spermatogenesis in concert with Nanos3
}

\author{
Manami SENOO ${ }^{1,2)}$, Hiroshi HOZOJI 1, 2), Yu ISHIKAWA-YAMAUCHI'), Takashi TAKIJIRI 1, 2), \\ Sho OHTA $^{3)}$, Tomoyo $\mathrm{UKAI}^{3)}$, Mio KABATA ${ }^{4)}$, Takuya YAMAMOTO ${ }^{4-7)}$, Yasuhiro YAMADA ${ }^{3)}$, \\ Masahito IKAWA ${ }^{1,8)}$ and Manabu OZAWA ${ }^{1)}$ \\ 1) Laboratory of Reproductive Systems Biology, Center for Experimental Medicine and Systems Biology, \\ The Institute of Medical Science, The University of Tokyo, Tokyo 108-8639, Japan \\ ${ }^{2)}$ Graduate School of Frontier Sciences, The University of Tokyo, Tokyo 108-8639, Japan \\ 3) Division of Stem Cell Pathology, Center for Experimental Medicine and Systems Biology, Institute of Medical Science, \\ University of Tokyo, Tokyo 108-8639, Japan \\ 4) Department of Life Science Frontiers, Center for iPS Cell Research and Application (CiRA), Kyoto University, \\ Kyoto 606-8507, Japan \\ 5) Institute for the Advanced Study of Human Biology (WPI-ASHBi), Kyoto University, Kyoto 606-8501, Japan \\ 6) AMED-CREST, Tokyo 100-0004, Japan \\ 7) Medical-risk Avoidance based on iPS Cells Team, RIKEN Center for Advanced Intelligence Project (AIP), \\ Kyoto 606-8507, Japan \\ ${ }^{8)}$ Research Institute for Microbial Diseases, Osaka University, Osaka 565-0871, Japan
}

\begin{abstract}
PTBP1, a well-conserved RNA-binding protein, regulates cellular development by tuning posttranscriptional mRNA modification such as alternative splicing (AS) or mRNA stabilization. We previously revealed that the loss of Ptbp 1 in spermatogonia causes the dysregulation of spermatogenesis, but the molecular mechanisms by which PTBP1 regulates spermatogonium homeostasis are unclear. In this study, changes of AS or transcriptome in Ptbpl-knockout (KO) germline stem cells (GSC), an in vitro model of proliferating spermatogonia, was determined by next generation sequencing. We identified more than 200 differentially expressed genes, as well as 85 genes with altered AS due to the loss of PTBP1. Surprisingly, no differentially expressed genes overlapped with different AS genes in Ptbpl-KO GSC. In addition, we observed that the mRNA expression of Nanos3, an essential gene for normal spermatogenesis, was significantly decreased in Ptbp 1-KO spermatogonia. We also revealed that PTBP1 protein binds to Nanos 3 mRNA in spermatogonia. Furthermore, Nanos $3^{+/} ; P_{t b p 1^{+/-}}$mice exhibited abnormal spermatogenesis, which resembled the effects of germ cell-specific Ptbpl KO, whereas no significant abnormality was observed in mice heterozygous for either gene alone. These data implied that PTBP1 regulates alternative splicing and transcriptome in spermatogonia under different molecular pathways, and contributes spermatogenesis, at least in part, in concert with NANOS3.
\end{abstract}

Key words: Alternative splicing, PTBP1, RNA-binding protein, Spermatogonia, Spermatogenesis

(J. Reprod. Dev. 66: 459-467, 2020)

n male mammals, spermatogenesis, a highly orchestrated process to produce mature sperm, is sustained throughout life under the strict control of various molecular mechanisms [1-3]. Recent advancements in sequencing technology have led to the discovery that testes rank among the top tissues concerning transcriptome complexity and alternative splicing (AS) [4-7]. AS changes RNA isoforms drastically

Received: May 10, 2020

Accepted: June 23, 2020

Advanced Epub:July 6, 2020

C2020 by the Society for Reproduction and Development

Correspondences: M Ozawa (e-mail: semil@ims.u-tokyo.ac.jp), M Ikawa (e-mail: ikawa@biken.osaka-u.ac.jp)

This is an open-access article distributed under the terms of the Creative Commons Attribution Non-Commercial No Derivatives (by-nc-nd) License. (CC-BY-NC-ND 4.0: https://creativecommons.org/licenses/by-nc-nd/4.0/) in accordance with testicular germ cell differentiation from mitotic spermatogonia to meiotic spermatocytes to haploid spermatids [4, 7]. AS is a mechanism for producing multiple gene products from a single gene locus using different combinations of coding or noncoding exons, thereby increasing the diversity of the transcriptome and proteome and facilitating proper tissue development $[8,9]$. In humans, more than $90 \%$ of genes have splicing isoforms [10].

RNA-binding proteins (RBPs) play a central role in posttranscriptional RNA modification such as AS or RNA stabilization and organizes tissue development and homeostasis $[6,11]$. The polypyrimidine tract-binding proteins (PTBPs) are highly conserved RBPs from Caenorhabditis elegans to mammals, and they have been implicated in many aspects of mRNA regulation including AS [12], polyadenylation [13], and stabilization [14]. In testes, two paralog genes of the PTBP family are expressed, e.g., PTBP1 and PTBP2, 
and these genes display stage-specific expression differences in testes. PTBP1 is mainly expressed by mitotic spermatogonia, and its expression is diminished by differentiation after the spermatocyte stage. Conversely, PTBP2 is hardly expressed by spermatogonia, whereas its expression becomes evident in meiotic spermatocytes [15]. Germ cell-specific loss of PTBP2 has little effect on transcriptomic regulation or AS in spermatogonia, whereas it drastically alters stage-specific changes in transcriptomic regulation or AS from spermatogonia to spermatocytes, resulting in the dysregulation of spermatogenesis after meiosis $[6,7]$. We previously developed a spermatogonium-specific Ptbpl conditional knockout model (Ptbpl cKO) and observed its phenotypic feature; that is, Ptbpl cKO animals exhibited an increased number of seminiferous tubules exhibiting degeneration of spermatogenesis accompanied by the slower proliferation of spermatogonia [16]. These previous results including ours suggest that the stage-specific regulation of the transcriptome or AS by PTBP family proteins plays an important role in normal spermatogenesis. However, the PTBP1-mediated regulation of the transcriptome or AS in spermatogonia has not been fully clarified.

In this study, we assessed the alteration of transcriptomic regulation and AS in cultured spermatogonia, i.e., germline stem cells (GSCs), lacking PTBP1 using next-generation sequencing (NGS). We demonstrated that 276 genes were differentially expressed in Ptbpl cKO GSCs compared with their control levels. In addition, 103 different AS isoforms of 85 genes were detected. It is notable that none of the differentially expressed genes overlapped with genes with different AS isoforms. We also observed using wildtype GSCs that PTBP1 protein binds to Nanos3, an essential factor for normal spermatogenesis [17], and Nanos 3 mRNA expression was decreased in Ptbp 1 cKO GSCs. Strikingly, mice heterozygous for both PTBP1 and NANOS3 exhibited an increased number of seminiferous tubules displaying degeneration of spermatogenesis, the genotypic features of which resemble germ cell-specific Ptbpl cKO mice [16]. Contrarily, heterozygous deletion of either gene alone did not result in significant abnormality of spermatogenesis. Collectively, our present data indicated an important role of PTBP1 in regulating the transcriptome and $\mathrm{AS}$ in spermatogonia and that the PTBP1-mediated regulation of spermatogenesis might act, at least in part, in concert with the function of NANOS3.

\section{Materials and Methods}

\section{Animals and ethics}

Mice were maintained under pathogen-free conditions in the experimental animal facility at the Institute of Medical Science of the University of Tokyo. All mouse experiments were approved by the Institutional Animal Care and Use Committee of the University of Tokyo (approval number PA17-64) and performed in compliance with their guidelines. C57BL/6J mice were purchased from Japan SLC (Shizuoka, Japan). Ptbp $1^{\text {flox }}$ and Ptbp $1^{+/-}$mice [18] as well as CAG-CreMer mice [19] were established in our laboratory via a conventional targeting strategy using 129-strain embryonic stem cells and backcrossed at least 10 times with C57BL/6J mice. Nanos3-Cre-pA (Nanos3 $3^{+/}$, ICR congenic) [17]

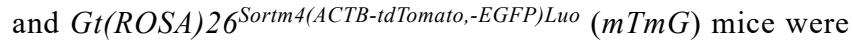
obtained from RIKEN BRC and the Jackson Laboratory, respectively.

\section{GSC development}

$m T m G ; C A G$-CreMer;Ptbp $1^{\text {flox/flox }}$ (Ptbp1-FF) or wild-type mice (BDF1 background) were used for GSC development according to a previously reported method [20]. In brief, the testes of 7-10-day-old postpartum neonates were collected and digested into single cells enzymatically. Single cells were seeded onto mitotically inactivated feeder cells (X-ray-irradiated or mitomycin C-treated mouse embryonic fibroblasts) in GSC culture medium with a slight modification. Specifically, $2 \mathrm{mM}$ GlutaMax and $5 \mathrm{mg} / \mathrm{ml}$ AlbuMax II (both from Thermo Fisher Scientific, Waltham, MA, USA) were added to the medium instead of $2 \mathrm{mM} \mathrm{L}$-glutamine and $5 \mathrm{mg} / \mathrm{ml}$ bovine serum albumin, respectively [21], and cells were cultured at $37^{\circ} \mathrm{C}$ in a humid atmosphere of $5 \% \mathrm{CO}_{2}$ to develop stably self-renewing GSCs. GSCs were passaged every $7-10$ days at a density of $2 \times 10^{5}$ cells/ $\mathrm{ml}$. To initiate the tamoxifen-induced deletion of the floxed allele in Ptbp1-FF GSCs, $1 \mu \mathrm{M}$ 4-hydroxitamoxifen (4OHT, LKT Laboratories, St. Paul, MN, USA) was added to the culture medium.

\section{RNA-seq}

Total RNA was extracted from GSCs using NucleoSpin RNA II or NucleoSpin RNA Plus (Macherey-Nagel, Düren, Germany) according to the manufacturer's instructions (DNase treatment was included in the RNA extraction procedure in this kit). RNA-seq libraries were generated using a Truseq Stranded mRNA samples prep kit (Illumina, San Diego, CA, USA). The resulting RNA-seq libraries were generated using single-ended sequencing at a length of $86 \mathrm{bp}$ on a NextSeq 500 (Illumina). RNA-seq reads were mapped to the mouse reference genome (mm10) using STAR version 2.5.3a with the GENCODE version M17 annotation gtf file after trimming adaptor sequences and bases with low-quality values using cutadapt version 1.16 [22]. Fragments per kilobase of exon per million mapped reads (FPKM) values were calculated as the expression levels of each gene using cufflinks-2.2.1 [23] with the mouse GENCODE (version M17, protein coding) annotation gtf file. For the splicing analysis, RNA-seq reads crossing exon-exon junctions were used to calculate inclusion ratios as described previously [24] and identify the alternatively spliced exons via statistical analysis (Fisher's exact test using a $2 \times 2$ table in which junction reads were divided by cell types, i.e.. control versus $\mathrm{cKO}$, and read types, i.e., inclusion versus exclusion) followed by multiple-test correction with a false discovery rate (Benjamini-Hochberg FDR) of less than 0.05 . The calculation of the inclusion ratio, the statistical analysis, and the clustering analysis were performed using R software with custom-made scripts. All the RNA-seq data are available in SRA (Bio- Project: PRJNA623420).

\section{Quantitative real-time PCR ( $q P C R)$ and reverse transcription PCR (RT-PCR)}

The primers used in this study are shown in Table 1. Total RNA was extracted from GSCs as described in the " $R N A$-seq" section. cDNA was synthesized using SuperScript VILO (Thermo Fisher Scientific) according to the manufacturer's instructions in a reaction volume of $10 \mu \mathrm{l}$. The synthesized cDNA was used for qPCR or RT-PCR. Taq DNA-polymerase Standard (Greiner Bio-One, Tokyo, Japan) was used for RT-PCR. Amplified DNA was visualized using Novel Juice (Bio-Helix, Keelung City, Taiwan, ROC), and signals were detected using BluPAD (LM, Tokyo, Japan). For qPCR analysis, 
Table 1. PCR primers

\begin{tabular}{clll}
\hline Method & \multicolumn{1}{c}{ Gene } & \multicolumn{1}{c}{ Forward } & \multicolumn{1}{c}{ Reverse } \\
\hline qPCR & Ptbpl & GGTCTCTTCCGTGTGCCATG & CTGCGCTCCTGTTGTCACCT \\
& Nanos3 & CACTACGGCCTAGGAGCTTGG & TGATCGCTGACAAGACTGTGGC \\
& Gapdh & ATGAATACGGCTACAGCAACAGG & CTCTTGCTCAGTGTCCTTGCTG \\
\hline RT-PCR & Cers5 & CCTACCCTTCCTGGTGGCTCTTC & GGTTGCCAGTGGCTTTACAAGTC \\
& Dpf 2 & AACCCGATGACTTCCTTGATGACC & TTTCTGCTCCTCAGACCTCTGG \\
& H13 & TCGGCTTTCCTGTCCTGGTG & AGAAAGGGCAGAGGGAACAGG \\
& Nanos 3 & GGCAAAGACACAGGATGCTGG & TGATCGCTGACAAGACTGTGGC \\
\hline
\end{tabular}

qPCR, quantitative real-time PCR; RT-PCR, reverse transcription PCR.

Thunderbird SYBR qPCR Mix (Toyobo, Osaka, Japan) and StepOne system (Thermo Fisher Scientific) were used for quantification. The fold difference was calculated using the $\Delta \Delta C_{t}$ method [25].

\section{Histological analysis}

Testes were fixed in PBS containing 4\% (v/v) paraformaldehyde (PBS-PFA) overnight at $4{ }^{\circ} \mathrm{C}$ and then dehydrated by serial treatment with gradient ethanol solutions (from 70 to $100 \%$ [v/v] ethanol). Thereafter, dehydrated testes were embedded in paraffin for sectioning at a thickness of $5 \mu \mathrm{m}$. For histological staining, sections were deparaffinized in Lemosol A (FUJIFILM Wako Pure Chemical, Tokyo, Japan), rehydrated by serial treatment with gradient ethanol solutions (from 100 to $70 \%[\mathrm{v} / \mathrm{v}]$ ethanol), washed in water, and stained with hematoxylin and eosin staining. Slides were covered by a coverslip with Mount-Quick mounting medium (Daido Sangyo, Tokyo, Japan) and observed using a microscope (BZ-X700, Keyence, Osaka, Japan).

\section{Immunohistochemistry}

For immunohistochemistry, testes were fixed in PBS-PFA overnight at $4{ }^{\circ} \mathrm{C}$, dehydrated, embedded in paraffin, and sectioned as described previously. Rehydrated sections were boiled in Tris-EDTA buffer (10 mM Tris, $1 \mathrm{mM}$ EDTA, and $0.05 \%$ [v/v] Tween-20 prepared in water, $\mathrm{pH} 9.0)$ via autoclaving $\left(120^{\circ} \mathrm{C}, 20 \mathrm{~min}\right)$ to reactivate antigens. Sections were then permeabilized with $0.3 \%(\mathrm{v} / \mathrm{v})$ Triton $\mathrm{X}$ diluted in Tris-buffered saline and blocked using $1 \%(\mathrm{w} / \mathrm{v})$ bovine serum albumin (Nacalai Tesque, Kyoto, Japan) and 10\% normal goat serum (Jackson ImmunoResearch, West Grove, PA, USA) at room temperature for $1 \mathrm{~h}$. Slides were incubated with primary antibodies (rabbit anti-PLZF (1:100, sc-22839, Santa Cruz Biotechnology, Dallas, TX, USA), anti-SCP3 (1:500, Santa Cruz), rat anti-germ cell-specific nuclear antigen (GENA; clone TRA98, BioAcademia, Osaka, Japan), or anti-TRA54 antibodies (1:100, Abcam, Cambridge, MA, USA)) at $4{ }^{\circ} \mathrm{C}$ overnight with gentle shaking. Then, sections were incubated with Alexa Fluor 488-, 555-, or 647-conjugated host species-specific secondary antibodies (all from Thermo Fisher Scientific) for $1 \mathrm{~h}$ at room temperature in the dark. After antibody exposure, sections were incubated with $0.1 \%$ (w/v) Sudan black B (Sigma-Aldrich, St. Louis, MO, USA) solution to quench autofluorescence signals. Then, sections were mounted on a coverslip with anti-fade mounting solution containing NucBlue nuclei staining dye (ProLong Glass; Thermo Fisher Scientific). Fluorescence signals were detected using a fluorescence microscope (BZ-X700).

\section{RNA immunoprecipitation (RNA-IP)}

RNA-IP using wild-type GSC lysate was performed using an RIP assay kit (MBL Life Science, Nagoya, Japan) according to the manufacturer's instructions. Mouse anti-PTBP1 (15 $\mu \mathrm{g}$ of antibody in $30 \mu \mathrm{l}$ of reaction mixture, \#324800, Thermo Fisher Scientific) or normal mouse IgG (negative control, \#5415S, Cell Signaling Technology, Danvers, MA, USA) was used for IP. Immunoprecipitated protein and RNA were analyzed using standard Western blotting or RT-PCR protocols, respectively. Protein bands were visualized using Luminata Forte (Merck Millipore, Tokyo, Japan), and signals were detected using the ChemiDoc Imaging System (Bio-Rad, Hercules, CA, USA).

\section{Statistical analysis}

All numerical data are presented as the mean \pm SEM. Differences between genotypes were tested using Student's $t$-test. P values less than 0.05 were considered significant.

\section{Results}

\section{PTBP1 is required for the regulation of AS in GSCs}

Undifferentiated spermatogonia can self-renew in culture via stimulation of GDNF and FGF2 while retaining their stem/progenitor characteristics [20]. We utilized this culture system to investigate whether PTBP1 regulates AS in spermatogonia by performing RNAseq. In this study, 4OHT was administered to induce the deletion of PTBP1 in Ptbp1-FF GSCs (Ptbpl cKO), and the solvent (ethanol)administered group was designated as the control. Ptbpl mRNA expression, which was visualized using Integrative Genomics Viewer (https://software.broadinstitute.org/software/igv/), was absent in Ptbpl cKO GSCs, whereas the mRNA expression of the adjacent Plppr 3 gene was comparable between control and Ptbp1 cKO GSCs, indicating that our inducible Ptbp $1 \mathrm{cKO}$ GSC model worked properly (Fig. 1A). Ptbpl cKO GSCs displayed severe growth defects in vitro (Fig. 1B), as we have reported previously [16]. RNA-seq reads, which spanned exon splice junction sites, were used to analyze the splicing pattern [26]. The pattern was categorized into six different types of AS, e.g., "skipped exon," "mutually exclusive exon," "alternative 5' splice site," "alternative 3' splice site," "alternative first exon," and "alternative last exon," by comparing the "inclusion ratio," which was defined as the ratio of the number of reads derived from the "inclusion (red-lined splicing)" junction region to the total number of 
(A)

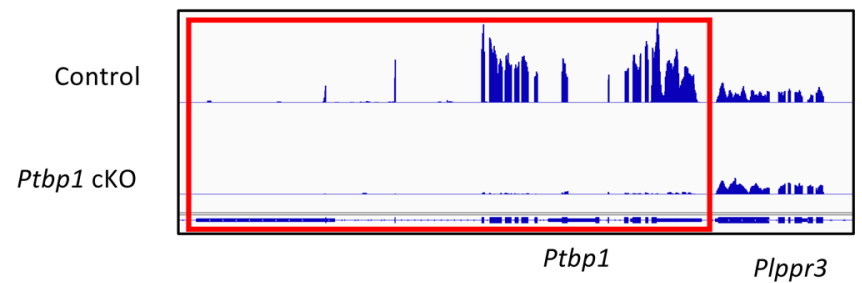

(B)

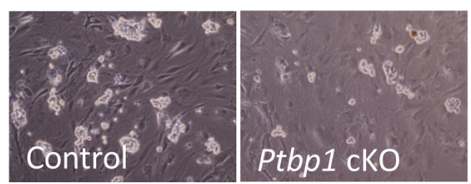

(C)
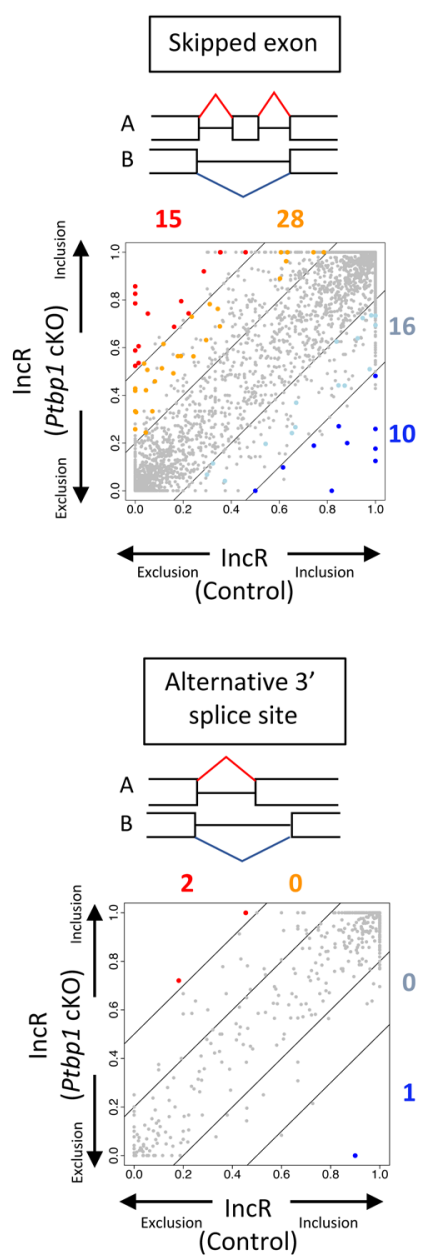

(D)

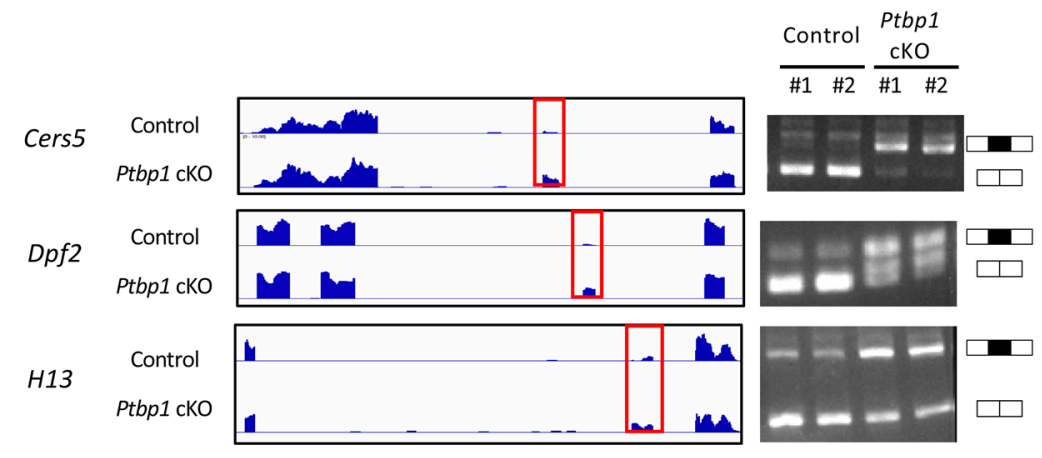

Fig. 1.
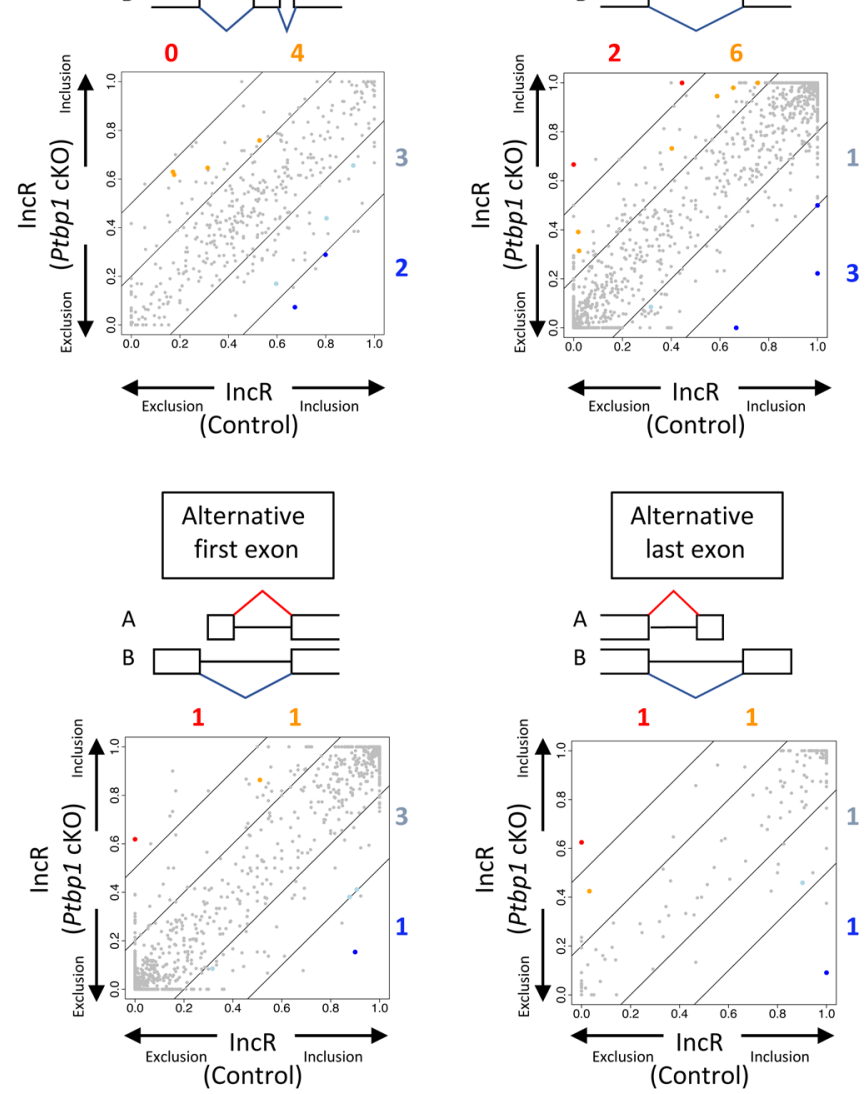
splice site

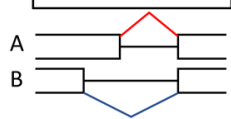

. 
reads derived from the inclusion and "exclusion (blue-lined splicing)" junction regions (Fig. 1C) as reported previously [24]. One hundred three different splicing events (in 85 genes) exhibited inclusion ratios that significantly differed (FDR $<0.05$ ) between control and Ptbpl cKO GSCs by a minimum absolute change of 0.2 (Fig. 1C). Three independent genes with different splicing isoforms as determined via RNA-seq analysis were selected to confirm the AS changes by RT-PCR (Fig. 1D). Notably, changes in "skipped exon" events, especially exon inclusion, were most evident after the deletion of PTBP1 in GSCs (Fig. 1C, left up panel). These observations implied that PTBP1 is required for AS in the spermatogonia and that the main role of PTBP1 in AS is to regulate cassette exon exclusion.

\section{Alteration of the transcriptome in Ptbp1-deleted GSC}

Next, we investigated the alteration of global mRNA expression patterns in Ptbpl cKO GSCs. By using $>2$ - or $<0.5$-fold changes as the cut-offs, a total of 276 genes displayed different expression levels (163 upregulated and 113 downregulated genes) between Ptbpl cKO and control GSCs (Fig. 2A). Interestingly, no differentially expressed genes between Ptbpl cKO and control GSCs exhibited featured different AS isoforms. We plotted the FPKM values of 85 differentially spliced genes, and they displayed strong concordance $(\mathrm{R}=0.97)$ between the Ptbp $1 \mathrm{cKO}$ and control GSCs (Fig. 2B), suggesting that PTBP1 controls either AS or mRNA expression, whereas the regulation machinery might be independent. To analyze which biological processes are relevant to the changes in the transcriptome of Ptbpl cKO GSCs, differentially expressed genes were examined via Gene Ontology (GO) analysis using Database for Annotation, Visualization and Integrated Discovery (DAVID; DAVID Bioinformatics Resources 6.8, https://david.ncifcrf.gov) [27]. Regarding genes upregulated in Ptbpl cKO GSCs, 12 GO terms were enriched (cut-off of $\mathrm{P}<$ 0.05 ), including two terms related to cell death or cell proliferation retardation, e.g., p53 signaling pathway and regulation of apoptotic process (Fig. 2C). The term 'p53 signaling pathway' contained Casp8, Bcl3, Pax8, Card11, Agtr2, and Phlpp1, and the term 'regulation of apoptotic process' contained Perp, Adgrb1, Steap3, and Casp8. Concerning genes downregulated in Ptbpl cKO GSCs, nine GO terms were listed. Interestingly, Nanos3, which is known as an essential factor for germ cell development or spermatogenesis [28, 29], was included under the GO terms 'cell differentiation' and 'multicellular organism development' (Fig. 2D).

\section{PTBP1 protein binds to Nanos3 $m R N A$}

Our RNA-seq analysis revealed that Nanos 3 mRNA expression is downregulated in Ptbpl cKO GSCs compared with the control levels (Fig. 3A). Thus, we next analyzed to confirm Nanos 3 mRNA reduction using qPCR. Nanos 3 mRNA expression was significantly decreased in Ptbpl cKO GSCs, and the extent of downregulation was comparable with that in RNA-seq analysis (Fig. 3B). PTPB1 is an RBP that regulates posttranscriptional RNA processing, such as AS and polyadenylation, to ensure RNA stability. Thus, we next attempted to confirm whether PTBP1 protein binds to Nanos 3 mRNA by RNAimmunoprecipitation (RNA-IP) using anti-PTBP1 antibody followed by RT-PCR. PTBP1 protein was specifically immunoprecipitated by anti-PTBP1 antibody (Fig. 3C), and Nanos 3 mRNA was detected in the anti-PTBP1-immunoprecipitated ribonucleoprotein samples (Fig. 3D). These results clearly demonstrated that PTBP1 binds to Nanos 3 mRNA, thus indicating the co-regulatory roles of PTBP1 and NANOS3 in spermatogonia.

\section{Ptbp1 and Nanos3 double heterozygous deletion results in abnormal spermatogenesis in mice}

The results from the present analysis led us to hypothesize that PTBP1 maintains the proliferation of spermatogonia in concert with NANOS3. Our previous paper reported that approximately $20 \%$ of seminiferous tubules display degeneration of spermatogenesis, i.e., tubules with no germ cells, in spermatogonium-specific Ptbp $1 \mathrm{cKO}$ models [16]. Conversely, conventional Ptbpl knockout (KO) is embryonically lethal around gastrulation [29], and conventional Nanos3 $\mathrm{KO}$ results in complete infertility because of a defect in primordial germ cell development [17]. Therefore, we developed the Ptbp 1 and Nanos 3 double heterozygous deletion mouse $\left(\right.$ Nanos $^{+/-} ;$Ptbp $\left.^{+/-}\right)$ to assess the roles of these genes in spermatogenesis. Weight of the testis did not differ significantly at 5 weeks old (Fig. 4A). At that age, almost all seminiferous tubules exhibited normal spermatogenesis in the $\mathrm{Nanos}^{+/-}$or Ptbp $1^{+/-}$testis. By contrast, significantly more seminiferous tubules exhibited degeneration of spermatogenesis in the Nanos $3^{+/-} ;$Ptbp $1^{+/-}$testis (Fig. 4B, asterisk). Immunohistochemistry revealed that many degenerated seminiferous tubules did not contain germ cells including spermatogonia ( $\mathrm{PLZF}+$ ), spermatocytes $(\mathrm{SCP} 3+)$, and spermatids (TRA54+, Fig. 4C). The proportions of seminiferous tubules exhibiting degeneration of spermatogenesis were less than $1 \%$ in three of four $\mathrm{Nanos}^{+/-}$mice and all four $\mathrm{Ptbp1}^{+/-}$mice, and the percentage was less than $2.5 \%$ in the fourth $\mathrm{Nanos}^{+/-}$mouse. Conversely, the ratios exceeded $5 \%$ in three of five $\mathrm{Nanos}^{+/-} ; \mathrm{Ptbp}^{+/-}$ mice, with the highest proportion being $31.1 \%$ in one mouse (Fig. 4D). These results suggest that the additive effect of PTBP1 and NANOS3 plays an important role in normal spermatogenesis.

\section{Discussion}

In this study, we examined the roles of PTBP1, a highly conserved RBP that is strongly expressed by spermatogonia, in global AS and transcriptomic regulation in spermatogonia. For this purpose, we conducted RNA-seq analysis using Ptbpl cKO GSCs and identified more than 200 differentially expressed genes, as well as 85 genes

Fig. 1. Polypyrimidine tract-binding protein 1 (PTBP1) is required for regulation of alternative splicing in germline stem cells (GSCs). (A) RNA-seq reads revealing the loss of Ptbpl mRNA expression in Ptbpl conditional knockout (cKO) GSCs. (B) Representative morphology of control (upper panel) and Ptbpl cKO GSCs (lower panel). (C) Scatter plots present the inclusion ratios of Ptbpl cKO and control GSCs. Each dot indicates a splicing event whose inclusion ratio was changed by more than 0.5 -fold (red and blue) or $0.2-0.5$-fold (orange and light-blue) (Fisher's exact test, false discovery rate $<0.01$ ). The numbers of the dot in color in each fraction are shown. (D) RNA-seq reads of Cers5, $\mathrm{Dpf}$, and $\mathrm{H} 13$ revealing that alternative exons are highly retained in Ptbpl cKO GSCs (left panels). Different alternative splicing patterns were confirmed by reverse transcription-PCR (right panels). 
(A)

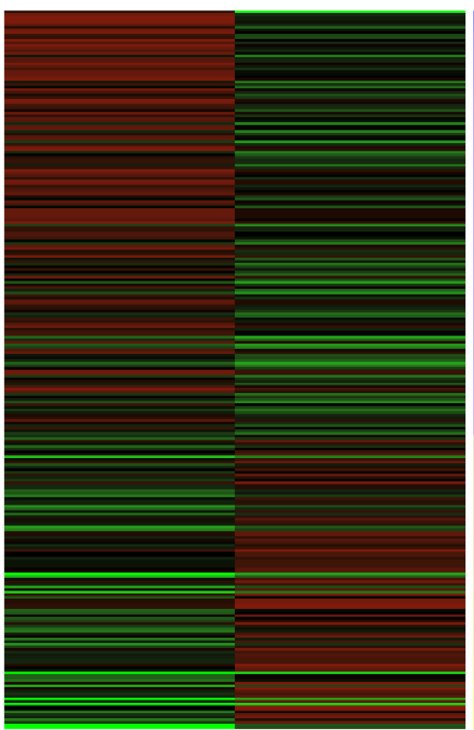

Control

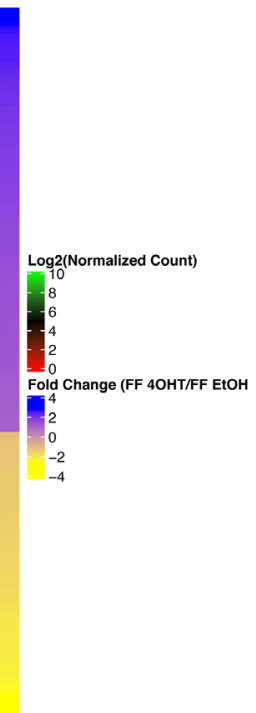

Ptbp1 cKO
(B)

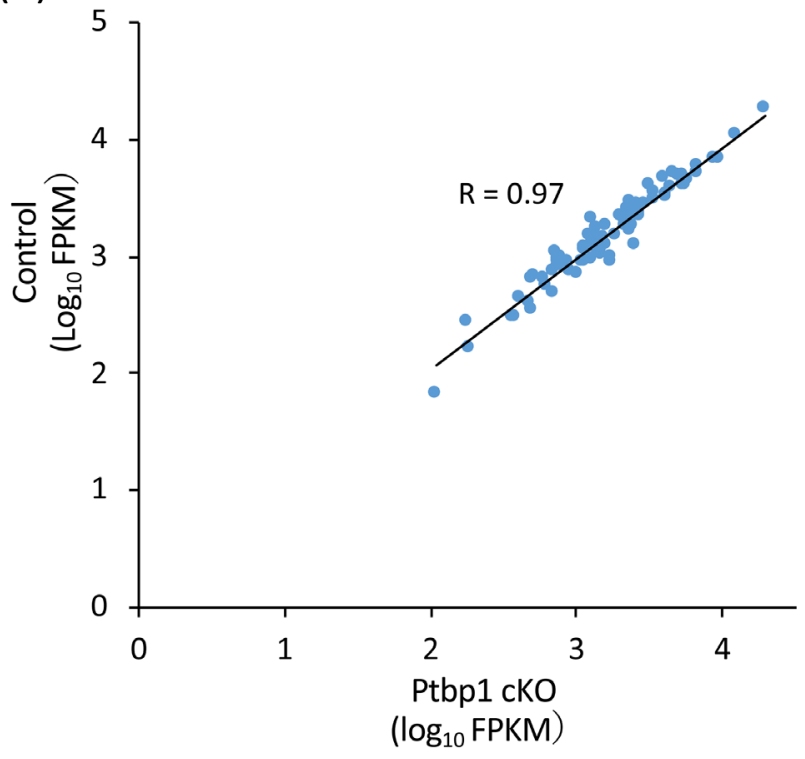

\section{>2-fold Upregulated}

locomotory behavior morphine addiction positive regulation of transcription from RNA polymerase II promoter central nervous system development proteolysis adrenergic signaling in cardiomyocytes axon guidance fat cell differentiation p53 signaling pathway regulation of apoptotic process positive regulation of transcription, DNA-templated axon guidance 0

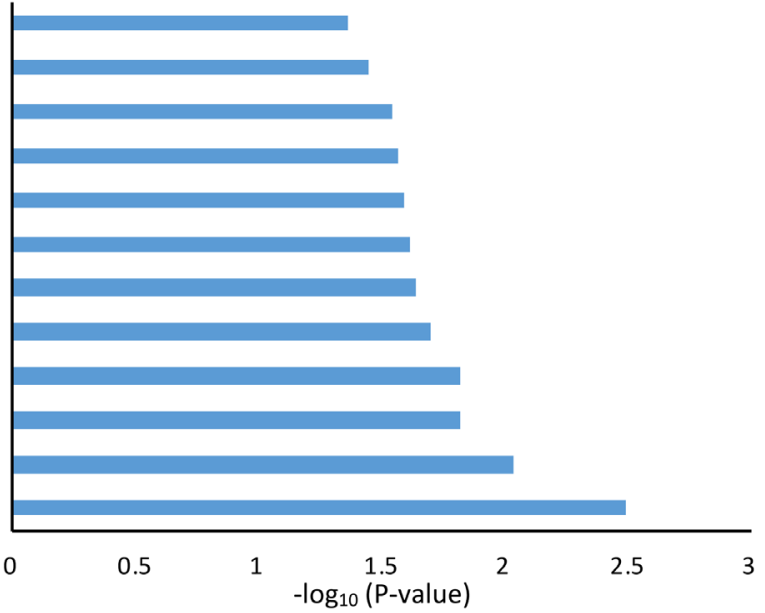

(D)

\section{$>2$-fold Downregulated}

negative regulation of transcription from RNA polymerase II promoter regulation of transcription, DNA-templated positive regulation of transcription from RNA polymerase II promote transcription, DNA-templated lipid metabolic process multicellular organism development cell differentiatio negative regulation of transcription, DNA-templated lipid catabolic proces

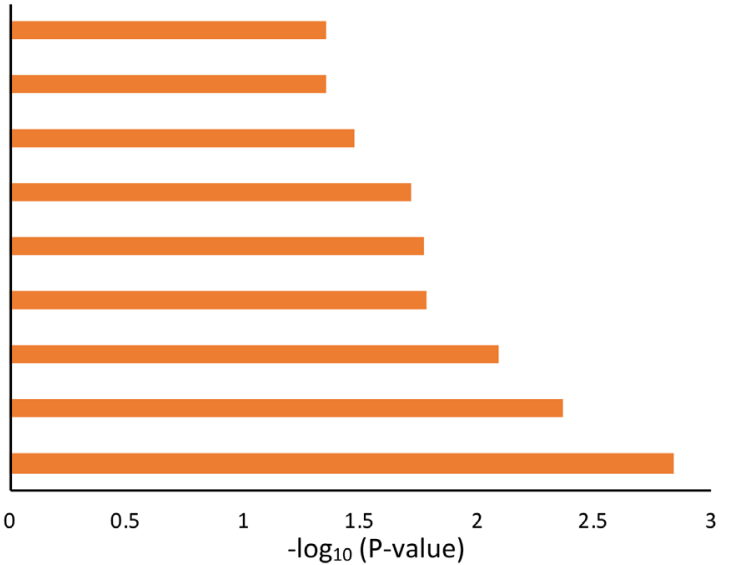

Fig. 2. 
(A)
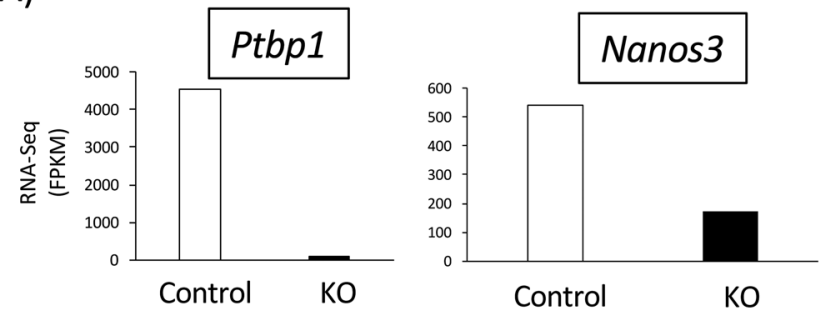

(B)

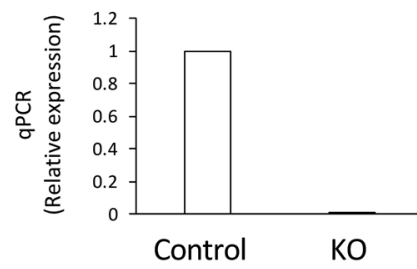

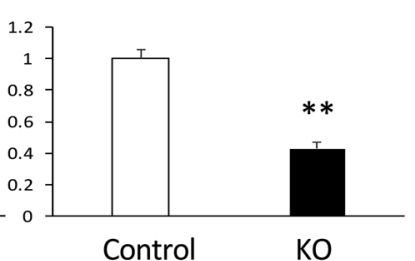

(C)

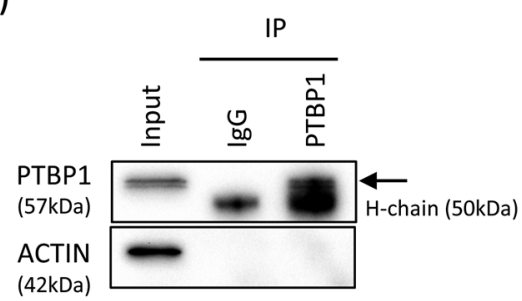

(D)

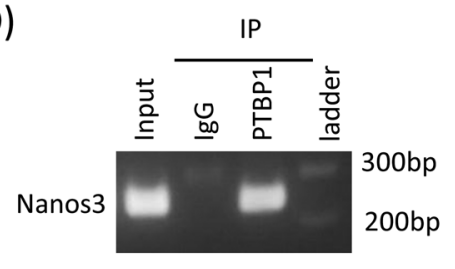

Fig. 3. Polypyrimidine tract-binding protein 1 (PTBP1) protein binds to Nanos3 mRNA. (A) Fragments per kilobase of exon per million mapped reads (FPKM) values of Ptbpl and Nanos 3 are shown in the left and right panels, respectively. (B) The reduction of Nanos 3 mRNA expression in Ptbp 1 conditional knockout germline stem cells (GSCs) was also confirmed by quantitative real-time PCR. Asterisks indicate a significant difference (** $\mathrm{P}<0.01 ; \mathrm{n}=3$ ). (C) Immunoblotting of PTBP1 (upper panel) or pan-ACTIN (bottom panel) following immunoprecipitation by an antiPTBP1 antibody or IgG-conjugated microbeads using wild-type GSC lysate. (D) mRNA was extracted from anti-PTBP1-immunoprecipitated GSC lysate and analyzed by reverse transcription-PCR using a Nanos3-specific primer set.

with altered AS due to the loss of PTBP1. We also confirmed that PTBP1 protein binds to Nanos 3 mRNA, an essential gene for normal spermatogenesis [17], and its mRNA expression is downregulated in Ptbp 1 cKO GSCs. In addition, mice featuring heterozygous deletion of both Ptbpl and Nanos $3\left(\right.$ Nanos $^{3^{+-}} ;$Ptbp $\left.^{+-}\right)$exhibits an increased number of seminiferous tubules displaying degeneration of spermatogenesis, in line with the findings in germ cell-specific Ptbpl cKO mice, whereas mice with heterozygous for either gene alone $\left(\mathrm{Nanos}^{+/-}\right.$or $\mathrm{Ptbp}^{+/-}$) did not exhibit significant abnormal spermatogenesis. Taken together, our present data indicate the important roles of PTBP1 in transcriptomic regulation and AS in spermatogonia. Furthermore, although further analysis to determine PTBP1 or NANOS3 expressions in the $\mathrm{Nanos}^{+/-}$; $\mathrm{Ptbp}^{+/-}$spermatogonia will be needed in the future experiment, the present results imply that PTBP1 regulates spermatogenesis, at least in part, in concert with NANOS3 function.

PTBP1 is a highly conserved RBP that is known to control cellular differentiation or specification by regulating the posttranscriptional modification of immature mRNAs. For example, PTBP1 governs the differentiation of neural progenitor cells to ependymal cells [30] or neurons by regulating AS of cell type-specific exons [31]. PTBP1 is also essential for the differentiation of immature B-cells to antigen-producing mature B-cells [32]. Our recent data using germ cell-specific Ptbpl cKO uncovered that this gene plays an important role in normal spermatogenesis, especially in the proliferation of spermatogonia [16]. This is in agreement with our present findings that the deletion of Ptbpl from cultured GSCs results in a drastic decrease in cell proliferation.

PTBP1 is known to regulate AS mainly via exon skipping by binding to the polypyrimidine-rich intronic region of nascent RNAs [33]. Along these lines, we detected that 'exon skipping' was most commonly altered in Ptbpl cKO GSCs. Interestingly, the expression of genes with different AS isoforms in Ptbpl cKO GSC was comparable with that in the control cells, and the correlation was strong $(\mathrm{R}=$ 0.97). In addition, no genes featuring AS isoforms overlapped with differentially expressed genes between Ptbpl cKO and control GSCs. Germ cell-specific cKO of PTBP2, a homolog of PTBP1 with highly overlapping target genes [34], also resulted in drastic changes in the AS isoforms of a number of genes in the testes, whereas the FPKM values of the different AS genes were comparable with high correlation rates $\left(\mathrm{R}^{2}=0.93\right)$ with the findings in the WT control [7]. Therefore, PTBP1 might govern AS or transcriptomic regulation under different molecular pathways.

NANOS3 is another RBP that has essential roles in spermatogenesis; e.g., NANOS3 regulates primordial germ cell (PGC) development

Fig. 2. Alteration of transcriptome in polypyrimidine tract-binding protein 1 (Ptbpl)-deleted germline stem cells (GSCs). (A) Heatmap revealing the levels of differentially expressed genes (276 genes) in Ptbpl conditional knockout (cKO) GSCs. (B) Comparison of fragments per kilobase of exon per million mapped reads (FPKM) values for the 85 genes with significantly different alternative splicing isoforms between $P t b p 1 \mathrm{cKO}$ and control GSCs. (C and D) Gene Ontology (GO) analysis of upregulated (C) and downregulated genes (D) in Ptbp1 cKO GSCs (cut-off of 2-fold). DAVID Bioinformatics Resources 6.8 was used for the analysis, and significantly enriched GO terms are listed $(\mathrm{P}<0.05)$. 
(A)

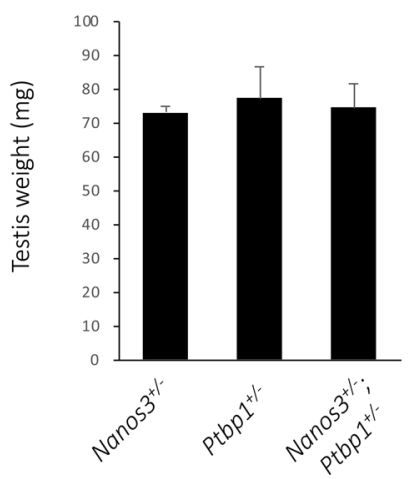

(C)
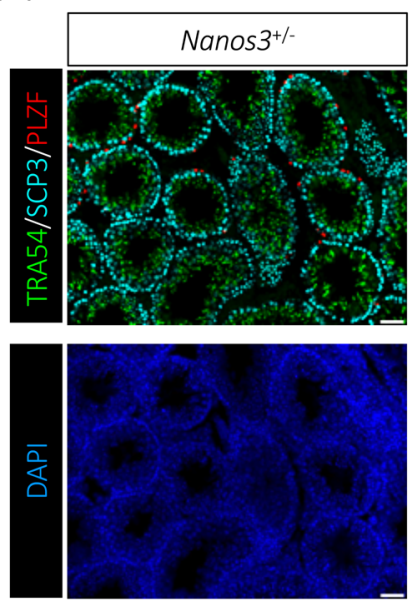

(B)

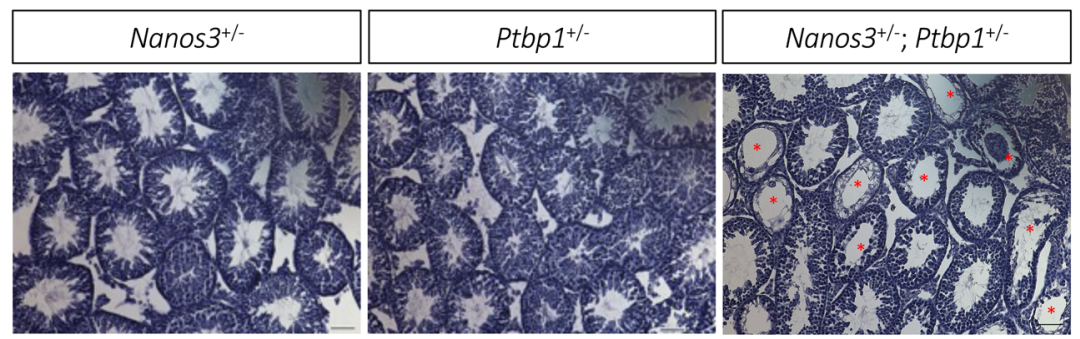

(D)
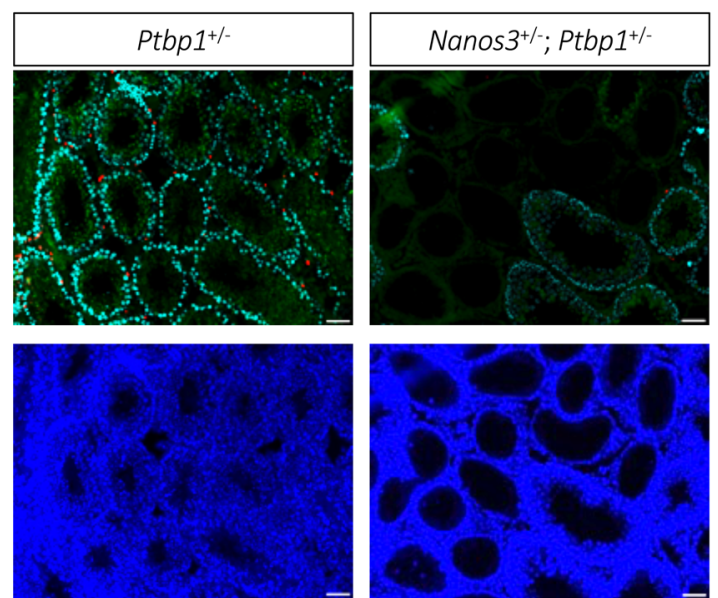

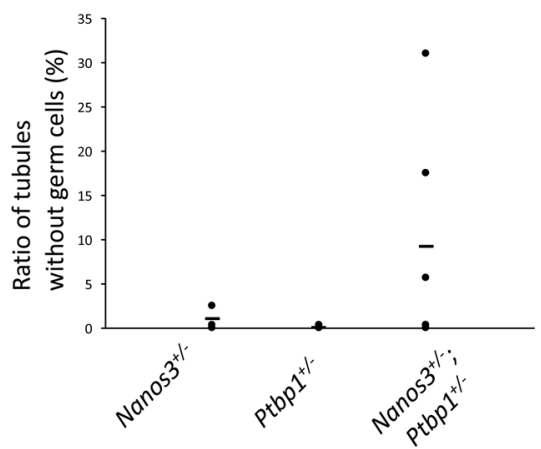

Fig. 4. Heterozygous deletion of both polypyrimidine tract-binding protein 1 (Ptbp1) and Nanos 3 results in abnormal spermatogenesis in mice. (A) Testis weight of mice in each genotype at 5-week of age $\left(\operatorname{Nanos}_{3}{ }^{+/}\right.$and $\operatorname{Ptbp~}^{+/-}, \mathrm{n}=4 ; \operatorname{Nanos}^{+/-} ;$Ptbp $\left.^{+/-}, \mathrm{n}=5\right)$. (B) Hematoxylin and eosin staining of testes from $\mathrm{Nanos}^{+/-}$(left), PtbpI ${ }^{+/-}$(middle), and $\mathrm{Nanos}^{++-} ; \mathrm{Ptbp1}^{+/-}$mice (right). The asterisk denotes seminiferous tubules exhibiting degeneration of spermatogenesis. Scale bar denotes $100 \mu \mathrm{m}$. (C) Immunohistochemical analysis of the testicular germ cell distribution in different stages of spermatogenesis in $\operatorname{Nanos}^{3+-}$ (left), Ptbpl $^{+/-}$(middle), or $\operatorname{Nanos}^{+/-} ;$Ptbpl $^{+/-}$mice (right). Sections were stained with anti-PLZF (a marker of spermatogonia), anti-SCP3 (a marker of spermatocytes), and TRA54 (a marker of haploid spermatids). Nuclei were stained with NucBlue. Scale bar denotes $100 \mu \mathrm{m}$. (D) Ratio of seminiferous tubules displaying degeneration of spermatogenesis in each genotype. Each dot represents the number in each mouse, and the bar represents the average $\left(\operatorname{Nanos}_{3}^{+/-}\right.$and $P t b p 1^{+/-}, \mathrm{n}=4 ; \operatorname{Nanos}^{+/-} ;$Ptbp $\left.1^{+/-}, \mathrm{n}=5\right)$.

[17] or maintains cell cycles of spermatogonia [35], and male Nanos3 KO mice are infertile [17]. Nanos 3 mRNA is expressed by PGC as early as E9.5 embryo, then disappear after E14.5 [28]. Nanos3 expression is reactivated and maintained in spermatogonia after birth [28]. We observed that PTBP1 protein is expressed by PGC as early as E12.5 (data not shown), and fetal testis of PGC-specific Nanos3-Ptbp 1 double hetero deletion $\left(\mathrm{Nanos}^{+/ / \mathrm{Cr}-K I} ; \mathrm{Ptbp}^{+/ f f o x}\right.$ mouse in which floxed allele was removed only in PGC by Cre recombinase driven by Nanos 3 promoter) does not exhibit significant abnormality (Supplementary Fig. 1: online only). Therefore, it suggests that abnormal spermatogenesis observed in this study occurs after spermatogenesis begins.

Although the AS isoforms of Nanos 3 mRNA did not differ between Ptbp $1 \mathrm{cKO}$ and control GSCs, mRNA expression was significantly downregulated in Ptbpl cKO cells. Another important role of PTBP1 in addition to AS regulation is for regulating mRNA stability. For example, PTBP1 binds to 3'-untranslated region (UTR) of Cd154
mRNA [36, 37], or 5'-UTR of $A x l$ mRNA [38], and protects from immature degradation of mRNAs. Using wild-type GSC, we clearly found that PTBP1 binds to Nanos 3 mRNA, suggesting that PTBP1 has regulatory roles in Nanos 3 mRNA modification including maintenance of mRNA stability. Nanos $3 \mathrm{KO}$ resulted in a significant increase in apoptotic cell death in germ cells [17]. Increased apoptosis is also observed in Ptbpl cKO models [16], and the GO analysis in the present study revealed that the term 'regulation of apoptotic process' was significantly enriched in Ptbpl cKO GSCs. Strikingly, our in vivo $\mathrm{Nanos}^{+/-} ; \mathrm{Ptbp}^{+/-}$displayed an increase in the degeneration of spermatogenesis, and the phenotypic feature were similar to those of Ptbpl cKO mice [16], although intensity of the phenotype in $\mathrm{Nanos}^{++-} ; \mathrm{Ptbp}^{+/-}$varies among individual males. One possible explanation of this phenotypic variation is that mice used in this study have mixed genetic background. Nanos $3^{+/}$is ICR-backcrossed strain, and $P t b p 1^{+/-}$is F2-F4 generation of ICR-B6 mixed. We mated these

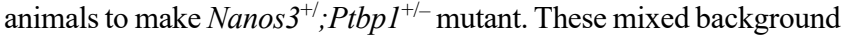


in each individual, especially in $\mathrm{Nanos}^{+/} ; \mathrm{Ptbp}^{+/-}$mutant, might be a cause of the variation. Collectively, although detailed mechanisms remain to be elucidated, the PTBP1-mediated regulation of spermatogenesis might occur, at least in part, in concert with NANOS3 function. Further study to determine relations of Ptbpl and Nanos3 on spermatogenesis, e.g., transgenic-rescue of Nanos 3 in the Ptbp 1 null spermatogonia will be needed.

\section{Acknowledgments}

Authors thank Dr N Yoshida and Dr H Sasanuma for their valuable advice and discussions, and $\mathrm{R}$ Sakamoto, M Kikuchi and $\mathrm{M}$ Baba for technical assistance. This work was supported by: the Ministry of Education, Culture, Sports, Science and Technology (MEXT)/Japan Society for the Promotion of Science (JSPS) KAKENHI grants (JP17K07132 and JP 20H03162 to MO); Grant-in-Aid for JSPS Fellows (JP19J01089) to YIY; Japan Agency for Medical Research and Development (AMED) grant (JP19gm5010001 to MI); Tokyo Biochemical Research Foundation grants to MI; and the Grant for Joint Research Project of the Research Institute for Microbial Diseases, Osaka University to MI, and MO.

\section{References}

1. de Rooij DG, Russell LD. All you wanted to know about spermatogonia but were afraid to ask. J Androl 2000; 21: 776-798. [Medline]

2. Oatley JM, Brinster RL. The germline stem cell niche unit in mammalian testes. Physiol Rev 2012; 92: 577-595. [Medline] [CrossRef]

3. McCarrey JR. Toward a more precise and informative nomenclature describing fetal and neonatal male germ cells in rodents. Biol Reprod 2013; 89: 47. [Medline] [CrossRef]

4. Schmid R, Grellscheid SN, Ehrmann I, Dalgliesh C, Danilenko M, Paronetto MP, Pedrotti S, Grellscheid D, Dixon RJ, Sette C, Eperon IC, Elliott DJ. The splicing landscape is globally reprogrammed during male meiosis. Nucleic Acids Res 2013; 41: 10170-10184. [Medline] [CrossRef]

5. Soumillon M, Necsulea A, Weier M, Brawand D, Zhang X, Gu H, Barthès P, Kokkinaki M, Nef S, Gnirke A, Dym M, de Massy B, Mikkelsen TS, Kaessmann H. Cellular source and mechanisms of high transcriptome complexity in the mammalian testis. Cell Reports 2013; 3: 2179-2190. [Medline] [CrossRef]

6. Zagore LL, Grabinski SE, Sweet TJ, Hannigan MM, Sramkoski RM, Li Q, Licatalosi DD. RNA binding protein Ptbp2 is essential for male germ cell development. Mol Cell Biol 2015; 35: 4030-4042. [Medline] [CrossRef]

7. Hannigan MM, Zagore LL, Licatalosi DD. Ptbp2 controls an alternative splicing network required for cell communication during spermatogenesis. Cell Reports 2017; 19: 2598-2612. [Medline] [CrossRef]

8. Kwan T, Benovoy D, Dias C, Gurd S, Provencher C, Beaulieu P, Hudson TJ, Sladek R, Majewski J. Genome-wide analysis of transcript isoform variation in humans. Nat Genet 2008; 40: 225-231. [Medline] [CrossRef]

9. Pan Q, Shai O, Lee LJ, Frey BJ, Blencowe BJ. Deep surveying of alternative splicing complexity in the human transcriptome by high-throughput sequencing. Nat Genet 2008; 40: 1413-1415. [Medline] [CrossRef]

10. Merkin J, Russell C, Chen $\mathbf{P}$, Burge $\mathbf{C B}$. Evolutionary dynamics of gene and isoform regulation in Mammalian tissues. Science 2012; 338: 1593-1599. [Medline] [CrossRef]

11. Fu XD, Ares M Jr. Context-dependent control of alternative splicing by RNA-binding proteins. Nat Rev Genet 2014; 15: 689-701. [Medline] [CrossRef]

12. Black DL. Mechanisms of alternative pre-messenger RNA splicing. Annu Rev Biochem 2003; 72: 291-336. [Medline] [CrossRef]

13. Castelo-Branco P, Furger A, Wollerton M, Smith C, Moreira A, Proudfoot N. Polypyrimidine tract binding protein modulates efficiency of polyadenylation. Mol Cell Biol 2004; 24: 4174-4183. [Medline] [CrossRef]

14. Knoch KP, Bergert H, Borgonovo B, Saeger HD, Altkrüger A, Verkade P, Solimena M. Polypyrimidine tract-binding protein promotes insulin secretory granule biogenesis. Nat Cell Biol 2004; 6: 207-214. [Medline] [CrossRef]

15. Xu M, Hecht NB. Polypyrimidine tract-binding protein 2 binds to selective, intronic messenger RNA and microRNA targets in the mouse testis. Biol Reprod 2011; 84: 435-439.
[Medline] [CrossRef]

16. Senoo M, Takijiri T, Yoshida N, Ozawa M, Ikawa M. PTBP1 contributes to spermatogenesis through regulation of proliferation in spermatogonia. J Reprod Dev 2019; 65 : 37-46. [Medline] [CrossRef]

17. Suzuki H, Tsuda M, Kiso M, Saga Y. Nanos3 maintains the germ cell lineage in the mouse by suppressing both Bax-dependent and -independent apoptotic pathways. Dev Biol 2008; 318: 133-142. [Medline] [CrossRef]

18. Shibayama M, Ohno S, Osaka T, Sakamoto R, Tokunaga A, Nakatake Y, Sato M, Yoshida N. Polypyrimidine tract-binding protein is essential for early mouse development and embryonic stem cell proliferation. FEBS J 2009; 276: 6658-6668. [Medline] [CrossRef]

19. Ichise H, Hori A, Shiozawa S, Kondo S, Kanegae Y, Saito I, Ichise T, Yoshida N. Establishment of a tamoxifen-inducible Cre-driver mouse strain for widespread and temporal genetic modification in adult mice. Exp Anim 2016; 65: 231-244. [Medline] [CrossRef]

20. Kanatsu-Shinohara M, Ogonuki N, Inoue K, Miki H, Ogura A, Toyokuni S, Shinohara T. Long-term proliferation in culture and germline transmission of mouse male germline stem cells. Biol Reprod 2003; 69: 612-616. [Medline] [CrossRef]

21. Ozawa M, Fukuda T, Sakamoto R, Honda H, Yoshida N. The histone demethylase FBXL10 regulates the proliferation of spermatogonia and ensures long-term sustainable spermatogenesis in mice. Biol Reprod 2016; 94: 92. [Medline] [CrossRef]

22. Martin M. Cutadapt removes adapter sequences from high-throughput sequencing reads. EMBnet Journal 2011; 17: 10.

23. Trapnell C, Williams BA, Pertea G, Mortazavi A, Kwan G, van Baren MJ, Salzberg SL, Wold BJ, Pachter L. Transcript assembly and quantification by RNA-Seq reveals unannotated transcripts and isoform switching during cell differentiation. Nat Biotechnol 2010; 28: 511-515. [Medline] [CrossRef]

24. Ohta S, Nishida E, Yamanaka S, Yamamoto T. Global splicing pattern reversion during somatic cell reprogramming. Cell Reports 2013; 5: 357-366. [Medline] [CrossRef]

25. Schmittgen TD, Livak KJ. Analyzing real-time PCR data by the comparative $C(T)$ method. Nat Protoc 2008; 3: 1101-1108. [Medline] [CrossRef]

26. Wang ET, Sandberg R, Luo S, Khrebtukova I, Zhang L, Mayr C, Kingsmore SF, Schroth GP, Burge CB. Alternative isoform regulation in human tissue transcriptomes. Nature 2008; 456: 470-476. [Medline] [CrossRef]

27. Huang W, Sherman BT, Lempicki RA. Bioinformatics enrichment tools: paths toward the comprehensive functional analysis of large gene lists. Nucleic Acids Res 2009; 37: 1-13. [Medline] [CrossRef]

28. Tsuda M, Sasaoka Y, Kiso M, Abe K, Haraguchi S, Kobayashi S, Saga Y. Conserved role of nanos proteins in germ cell development. Science 2003; 301: 1239-1241. [Medline] [CrossRef]

29. Suckale J, Wendling O, Masjkur J, Jäger M, Münster C, Anastassiadis K, Stewart AF, Solimena M. PTBP1 is required for embryonic development before gastrulation. PLoS One 2011; 6: e16992. [Medline] [CrossRef]

30. Shibasaki T, Tokunaga A, Sakamoto R, Sagara H, Noguchi S, Sasaoka T, Yoshida N. PTB deficiency causes the loss of adherens junctions in the dorsal telencephalon and leads to lethal hydrocephalus. Cereb Cortex 2013; 23: 1824-1835. [Medline] [CrossRef]

31. Zhang X, Chen MH, Wu X, Kodani A, Fan J, Doan R, Ozawa M, Ma J, Yoshida N, Reiter JF, Black DL, Kharchenko PV, Sharp PA, Walsh CA. Cell-type-specific alternative splicing governs cell fate in the developing cerebral cortex. Cell 2016; 166 1147-1162.e15. [Medline] [CrossRef]

32. Sasanuma H, Ozawa M, Yoshida N. RNA-binding protein Ptbp1 is essential for BCRmediated antibody production. Int Immunol 2019; 31: 157-166. [Medline] [CrossRef]

33. Sawicka K, Bushell M, Spriggs KA, Willis AE. Polypyrimidine-tract-binding protein: a multifunctional RNA-binding protein. Biochem Soc Trans 2008; 36: 641-647. [Medline] [CrossRef]

34. Vuong JK, Lin CH, Zhang M, Chen L, Black DL, Zheng S. PTBP1 and PTBP2 serve both specific and redundant functions in neuronal Pre-mRNA splicing. Cell Reports 2016; 17: 2766-2775. [Medline] [CrossRef]

35. Lolicato F, Marino R, Paronetto MP, Pellegrini M, Dolci S, Geremia R, Grimaldi P. Potential role of Nanos3 in maintaining the undifferentiated spermatogonia population. Dev Biol 2008; 313: 725-738. [Medline] [CrossRef]

36. Hamilton BJ, Genin A, Cron RQ, Rigby WFC. Delineation of a novel pathway that regulates CD154 (CD40 ligand) expression. Mol Cell Biol 2003; 23: 510-525. [Medline] [CrossRef]

37. Coles LS, Bartley MA, Bert A, Hunter J, Polyak S, Diamond P, Vadas MA, Goodall GJ. A multi-protein complex containing cold shock domain (Y-box) and polypyrimidine tract binding proteins forms on the vascular endothelial growth factor mRNA. Potential role in mRNA stabilization. Eur J Biochem 2004; 271: 648-660. [Medline] [CrossRef]

38. Cho CY, Chung SY, Lin S, Huang JS, Chen YL, Jiang SS, Cheng LC, Kuo TH, Lay JD, Yang YY, Lai GM, Chuang SE. PTBP1-mediated regulation of AXL mRNA stability plays a role in lung tumorigenesis. Sci Rep 2019; 9: 16922. [Medline] [CrossRef] 